\title{
Flexibility of Tolls for Optimal Flows in Networks with Fixed and Elastic Demands
}

\author{
Claude M. Penchina*
}

Department of Physics, University of Massachusetts at Amherst, Amherst, MA 01003 USA

Additional Affiliations: Department of Physics, King's College, Strand London WC2R-2LS, UK, ECE Department of UCSD, La Jolla CA 92093 USA, and Gilora Associates, Flemington, NJ, USA

\begin{abstract}
Tolls are used to influence users to "voluntarily" choose paths in the system which optimize flows (VSO) to minimize social costs. When the toll solutions are not unique, administrators gain the flexibility to vary tolls in order to meet other goals while still maintaining optimal flows. For Fixed-Demand networks, it was known that path-tolls can be adjusted. However, the link-toll solution was thought to be unique if the number of paths exceeds the number of links. We show, however, that link-toll solutions are non-unique even for many very large networks in which the number of paths greatly exceeds the number of links. Uniqueness of tolls under Elastic Demand is studied here for the first time in the literature. We examine the uniqueness (or lack thereof) of tolls needed to optimize flows with Elastic User Demand. We find that elastic demand eliminates the adjustability of path-toll solutions, and partially restricts the newly-found flexibility of link-tolls. These results should provide guidance for traffic network administrators in planning second-best toll policies for situations where marginal cost pricing may be politically or otherwise unpopular.
\end{abstract}

Keywords: Braess's paradox, elastic demand, fixed demand, link-tolls, path-tolls, uniqueness of tolls, wheatstone bridge.

\section{INTRODUCTION}

\subsection{Introduction: Background of Prior Work}

Traffic system administrators aim to diminish problems of congestion by using link-tolls or path-tolls to modify the user-equilibrium (UE) flow pattern, thereby replicating the system-optimized (SO) flow pattern for the same network. Tolls in multi-class-user transportation networks were studied by Dafermos [1]. However, only fixed user demand was considered. It was shown that the path toll solution is not unique; adding the same toll to each path does not change the flows. Clearly, if path tolls are increased sufficiently, the assumption of fixed demand eventually becomes a poor approximation to reality. However, similar studies for elastic demand are not found in the literature.

Dafermos provides a specific method to solve the linktoll problem, and shows that at least one solution exists. She states, without proof, that multiple solutions may exist in "relatively simple networks" where the number of paths is smaller than or comparable to the number of links. However, the solution provided "will be in general the only solution since usually the number of paths in a network greatly exceeds the number of links so that the number of equations ... is much greater than the number of unknowns". These statements have led to the rather strong but unproved statement that, except in "simple networks", "the link-toll pattern constructed above will be the only solution of the link-toll collection problem" [2], and even stronger statements that the link toll policy is unique [3].

\footnotetext{
*Address correspondence to this author at the Department of Physics, University of Massachusetts at Amherst, Amherst, MA 01003 USA; E-mail: Penchina@Physics.UMass.Edu, cmPenchina@GMail.com
}

\subsection{Introduction: Other Recent Work}

Three very recent works on networks with fixed demands showed examples of the non-uniqueness of optimal tolls. Dial $[4,5]$ provides algorithms to find Minimal Revenue Tolls. Yang and Lam [6] uses an EDO (equilibrium decomposition optimization) algorithm to find alternative tolls. Their solutions maintain the optimal flows. However, neither of these references found necessary and sufficient conditions under which solutions will actually differ from the Marginal Cost Tolls. Their numerical examples have more links than paths, so they are not suitable as new counter-examples to the uniqueness, since Dafermos [1] already showed such counter-examples for "simple" networks. Also, neither of them directly addressed correctly the effects of modified tolls on the flows under elastic demand (see Penchina [7]).

\subsection{Introduction: The Present Work}

Through consideration of several example networks, we study the question of uniqueness of the link-toll and path-toll solutions for general networks. We review and extend previous work for fixed user demand, and then further extend the treatment to include the more realistic and more interesting case of elastic demand, for which we find new and very different results.

In section 2, we discuss fixed demand. We conclude that there are non-unique link-toll solutions in wide classes of "complex" networks; i.e. networks in which the number of paths greatly exceeds the number of links. It is shown by counter-examples that the well known statement [1] about uniqueness of link-tolls is too limiting. This non-uniqueness is important for toll policy planning in networks, where linktolls are generally easier to collect than path-tolls. We show 
that for cases of fixed demand, link-toll planners often have some of the same flexibility as path-toll planners to adjust tolls (presumably for the social good) while still maintaining a system-optimized flow.

Although fixed demand is a very popular assumption in traffic theory, it is usually only an approximation to the more realistic and more complicated case of elastic demand. The voluntary-system-optimization (VSO) solution uses Marginal Cost Pricing to maximize the sum of User Surplus and Toll Revenue [8].

For elastic demand, the problem of adjusting toll policies while maintaining optimal system flows has not been studied before in the literature. In section 3, we show that elastic demand removes the well-known flexibility of setting pathtolls, and also reduces some of the newly found increased flexibility of setting link-tolls. It effectively reverses the roles of link-tolls and path-tolls in adjusting toll policies to simultaneously achieve system optimized flows and additional social goals.

\section{NON-UNIQUE LINK-TOLL SOLUTIONS (FIXED DEMAND)}

In this section we discuss several networks for which the link-toll solution is non-unique (i.e. changed tolls with unchanged flows) under conditions of fixed user demand. We do not presume to find necessary and sufficient conditions for the most general networks that have non-unique optimal tolls. However, we provide examples to show that, there is no limit to the size or complexity of the network in which the link toll solution is not unique. In section 2.6 , we show a specific procedure (algorithm) that both increases the size of a network, and increases the adjustability of the link toll policy, or even makes it adjustable when it was unique for the smaller original network.

For simplicity of the argument and notation, we use several examples to introduce networks with a single user class and fixed demand for each origin/destination (O/D) pair. Extension to multi-class-users is straightforward. It involves only added superscripts $\mathrm{i}=1,2, \ldots \mathrm{n}$, as in [1], and needs no further discussion here. Extension to elastic demand is more complicated, more interesting, and has some important effects; it is discussed in section 3. That discussion will make use again of all the examples in this section 2 .

We first illustrate five classes of networks with nonunique link-toll solutions. Some of these classes are more general than indicated by Dafermos [1]. We then introduce two more classes (combinations of the original five classes). Class 7, in particular can be quite "complex" in the sense of having many more paths than links, and serves as a good counterexample to claims of uniqueness of link tolls. Presumably, there are many more classes with non-unique linktolls than illustrated here, but we find that these seven are sufficient to elucidate the situation.

Although classes 1 - 3 do not have more paths than links, they are discussed because they, and also the examples from Dial $[4,5]$ and Yang and Lam [6], are useful as components of more complex classes.

\subsection{Fixed Demand - Network Class 1: Disjoint (Parallel)}

For each origin/destination (O/D) pair Oi / Dj, each path Pij,k $(\mathrm{k}=1,2, \ldots \mathrm{K})$ connecting $\mathrm{Oi}$ to $\mathrm{Dj}$ is independent of every other path. i.e. there are no intersections of paths except at Oi and Dj. These can be thought of as "parallel" paths, although a single isolated link is also a (trivial) member of this network class. No path shares a link or node with any other path. Changing link-tolls in ways that add the same arbitrary fixed toll Tij to every one of the $\mathrm{K}$ paths Pij,k connecting $\mathrm{Oi} / \mathrm{Dj}$ will not influence any user to move from one path to another. Therefore, there is considerable freedom to modify some link-tolls without changing the flow pattern. One example of this class is shown in Fig. (1, class 1).
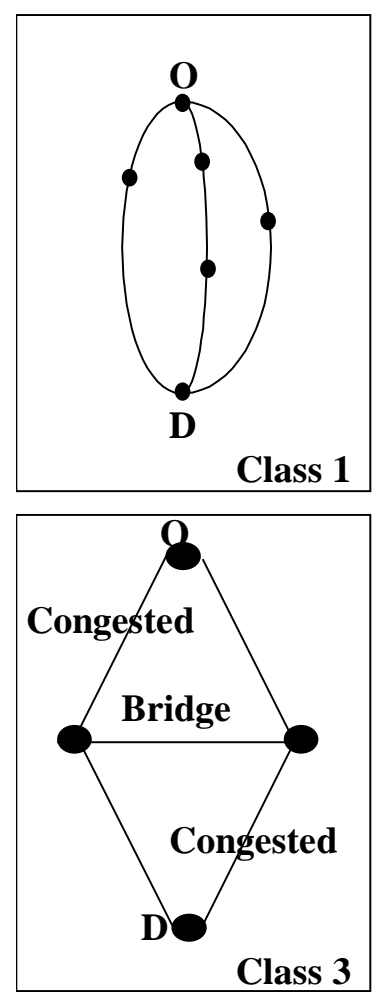


Fig. (1). Examples of Network Classes with Flexible Link Tolls.

Class $1=$ Disjoint $/$ Parallel.

Class $2=$ Fixed Number of Links per Path.

Class 3 = Bridge.

Class $4=$ Chain $/$ Series.

Class 5 = Concrete Example with Many More Paths than Links. 
This example is particularly simple, and seems somewhat trivial, in that one could divide any long link with one toll into a series of shorter links with their own individual tolls. However, more interesting uses of this case 1 are produced by combinations with other cases, as e.g. in section 2.6.

\subsection{Fixed Demand - Network Class 2: Fixed Number Of Links Per Path}

For each pair Oi/Dj, all paths Pij,k $(\mathrm{k}=1,2, \ldots \mathrm{K})$ from Oi to $\mathrm{Dj}$ have exactly the same (arbitrary positive integer) number $\mathrm{Nij}, \mathrm{k}=\mathrm{Nij}$ of links, independent of $\mathrm{k}$. However, it is not necessary that $\mathrm{Nij}=\mathrm{Ni}^{\prime} \mathrm{j}$ ' for any other pair $\mathrm{Oi}^{\prime} / \mathrm{Dj}$ '. Any two or more paths may share one or more links. One example of this class is shown in Fig. (1, class 2).

Given any link-toll policy which renders a user-equilibrium (UE) flow pattern system-optimizing (SO), the addition of the same arbitrary toll $\mathrm{T}$ to the existing toll on each link in this network will increase the path-toll by the same amount $\mathrm{T}$ $\mathrm{x}$ Nij on each path from Oi to $\mathrm{Dj}$. This added toll maintains the equality of costs on alternate path choices, and maintains the system-optimizing flow.

\subsection{Fixed Demand - Network Class 3: Bridge}

This class consists of bridged networks, such as the one in the Braess paradox. When all traffic uses the bridge (transversal link), a single toll on the bridge can replace two smaller tolls on each of the two congested links without changing any flows (see Penchina [9]. An example of this network class is shown in (Fig. 1, class 3 ).

\subsection{Fixed Demand - Network Class 4: Chain (Series)}

This class consists of a serial chain made of networks of other classes. It has at least the link-toll flexibility of each of the sub-networks. If the same network is repeated, it can be thought of as a "super-link" in a linear chain, or a "monomer" in a polymer (see Szwarc [10]), etc. A simple but interesting example uses the 3-link, 2-path network of Fig. (1, class 4 ) as the monomer repeated $\mathrm{M}$ times in series to form a linear polymer, with a single origin at the beginning, and a single destination at the end of the series.
For large $\mathrm{M}$, the $2^{\mathrm{M}}=\exp (\mathrm{M} \ln (2))$ paths exponentially exceed the $3 \mathrm{M}$ links. Still, the link-toll policy is not unique. Wherever only one link connects two nodes, that link-toll may be adjusted at will, since the user can not change paths. Wherever two links connect two nodes, addition of the same arbitrary (positive or negative) toll to each link does not disturb the equality of path costs, and will not change flow patterns. This flexibility of link-tolls is present independently at each monomer in the polymer chain. This is a clear and strong counterexample to the claim of Dafermos that the reason for the existence of many solutions is that the number of allowed paths is comparable to [1] or less than [1] the number of links, since the number of paths is exponentially larger than the number of links.

\subsection{Fixed Demand - Network Class 5: Concrete Example with More Paths than Links}

Fig. (1, class 5) shows a fairly small concrete example of a "complex" network, with many more paths, 13, than links, 5 , for which the link-toll pattern is non-unique; another clear counter-example to the uniqueness claim [1]. Here, the linkcost functions $\mathrm{Ci}$ (fi) $=$ hi + gi fi depend only on the local link flow fi, where hi is a fixed link cost (overhead cost) and gi is the non-negative incremental cost per unit of flow on that link due to congestion (congestion cost). Table $\mathbf{1}$ has more specifications and results of a detailed numerical example showing various possible solutions.

Note in Table 1, that the Marginal Cost solution, which induces system-optimal link flows, imposes tolls on all five links. In our example, it collects a high total revenue (comparable to the toll-free user costs). Clearly, there is no flowpattern-related reason to impose a toll on link $\mathrm{c}$, since this toll can not influence users to change links. Nor is there any such reason to use tolls on both links a and b (or both links $d$ and e) since only the difference between the tolls on two parallel links influences the user to choose between them. Thus, the optimal link-toll solution is surely not unique in this case.

The "simplest" solution, in the sense of having the smallest number of non-negative tolls, induces a system-optimized flow pattern with only two tolls. (This solution shown in

Table 1. User Equilibrium and System-Optimized Link-Toll Solutions for Network Class 5 with Fixed O/D Demands: $d_{X Y}=3 d_{W Z}$ $=4 d_{W X}=d_{Y Z}=d_{W Y}=d_{X Z}=2$

\begin{tabular}{|c|c|c|c|c|c|c|c|c|c|c|c|c|c|}
\hline \multirow[b]{2}{*}{ Link } & \multirow{2}{*}{$\begin{array}{c}\text { Link Cost } \\
\text { Ci(fi) }\end{array}$} & \multicolumn{3}{|c|}{ User Equilibrium. No Tolls } & \multicolumn{3}{|c|}{ System Optimal Flows } & \multicolumn{2}{|c|}{ Marginal Cost Toll } & \multicolumn{2}{|c|}{ Simple Toll* } & \multicolumn{2}{|c|}{ Fair Toll** } \\
\hline & & $\begin{array}{c}\text { Link } \\
\text { flow f }\end{array}$ & $\begin{array}{c}\text { Link } \\
\text { Costs } \\
\text { Per } \\
\text { User }\end{array}$ & $\begin{array}{l}\text { Total } \\
\text { Link } \\
\text { User } \\
\text { Cost }\end{array}$ & $\begin{array}{c}\text { Link } \\
\text { Flow f }\end{array}$ & $\begin{array}{c}\text { Link } \\
\text { Cost } \\
\text { Per User } \\
\text { w/o Toll }\end{array}$ & $\begin{array}{l}\text { Total } \\
\text { Link } \\
\text { User } \\
\text { Costs }\end{array}$ & $\begin{array}{c}\text { Link } \\
\text { Toll T }\end{array}$ & $\begin{array}{c}\text { Link } \\
\text { Revenue }\end{array}$ & $\begin{array}{c}\text { Link } \\
\text { Toll T }\end{array}$ & $\begin{array}{c}\text { Link } \\
\text { Revenue }\end{array}$ & $\begin{array}{c}\text { Link } \\
\text { Toll T }\end{array}$ & $\begin{array}{c}\text { Link } \\
\text { Revenue }\end{array}$ \\
\hline a & $4+3 \mathrm{fa}$ & $\mathrm{fa}=04$ & $\mathrm{Ca}=16$ & 064 & $\mathrm{fa}=03$ & $\mathrm{Ca}=13$ & 039 & 09 & 027 & 4 & 12 & +3 & +9 \\
\hline $\mathrm{b}$ & $12+\mathrm{fb}$ & $\mathrm{fb}=04$ & $\mathrm{Cb}=16$ & 064 & $\mathrm{fb}=05$ & $\mathrm{Cb}=17$ & 085 & 05 & 025 & 0 & 00 & -1 & -5 \\
\hline $\mathrm{c}$ & $1+4 \mathrm{fc}$ & $\mathrm{fc}=11$ & $\mathrm{Cc}=45$ & 495 & $\mathrm{fc}=11$ & $\mathrm{Cc}=45$ & 495 & 44 & 484 & 0 & 00 & $=0$ & $=0$ \\
\hline d & $4+3 f d$ & $\mathrm{fd}=04$ & $\mathrm{Cd}=16$ & 064 & $\mathrm{fd}=03$ & $\mathrm{Cd}=13$ & 039 & 09 & 027 & 4 & 12 & +3 & +9 \\
\hline $\mathrm{e}$ & $12+\mathrm{fe}$ & $\mathrm{fe}=04$ & $\mathrm{Ce}=16$ & $\underline{064}$ & $\mathrm{fe}=05$ & $\mathrm{Ce}=17$ & $\underline{085}$ & 05 & 025 & 0 & 00 & -1 & -5 \\
\hline & TOTAL & & & 751 & & & 743 & & 588 & & 24 & & +8 \\
\hline
\end{tabular}

*The "Simplest" Toll, in the sense of achieving VSO with the minimum number of non-negative tolls. It turns out to be the so-called "Minimal-Revenue" Toll [4, 5].

**The Fair Toll [1] makes the VSO link cost per user equal to user equilibrium link cost per user. It includes negative tolls (i.e. subsidies). 
Table 1 turns out to be an example of what Dial [4] called a Minimal Revenue solution.) To accomplish this, impose the first toll on that link (of links a and b) with the lower user marginal-cost in order to equalize the two marginal costs; and the second toll in the same way on links $d$ and e.

Also shown in Table $\mathbf{1}$ is the so-called "Fair" solution suggested by Dafermos [1] for which the user costs are the same as for user-equilibrium (UE) without tolls. This requires tolls on four links, two of them being negative tolls (subsidies). Note that the "Fair" solution has even lower net revenue than the so-called MR solution which does not allow subsidies.

\subsection{Fixed Demand - Network Class 6: Combination of 1 \& 2}

This class combines classes 1 and 2 . Each of the $\mathrm{K}$ disjoint paths $\mathrm{Pij}, \mathrm{k}(\mathrm{k}=1,2, \ldots, \mathrm{K})$ from Oi to $\mathrm{Dj}$ in Class 1 is replaced by a group of $\mathrm{M}(\mathrm{k})$ paths of Class $2, \mathrm{Pij}, \mathrm{km}(\mathrm{m}=1$, $2, \ldots, M(k))$, each of which has the same number Nij,k of links, independent of $\mathrm{m}$. Here, for example, one can modify the link-tolls in such a way that the change in each link toll in the group of $\mathrm{M}(\mathrm{k})$ paths $\mathrm{Pij}, \mathrm{km}$ is the same arbitrary fixed number $\mathrm{Cij}, \mathrm{k}=\mathrm{Cij} / \mathrm{Nij}, \mathrm{k}$, independent of $\mathrm{m}$. This will change the cost on each path Pij,km by a fixed amount $\mathrm{Cij}$, independent of $\mathrm{k}$ or $\mathrm{m}$, and will not change the flow pattern.

\subsection{Fixed Demand - Network Class 7: Compound}

Given any complex network, one can add any one of the above "simpler" networks, or even a single isolated link, to an outer node of the complex network. This new "compound network" (= complex + simple network), larger, and in some ways even more complex than the starting complex network, has at least all the link-toll flexibility of the added simpler network. It is a good counter-example to the claim [1] of unique link-toll solutions in general large networks.

It seems likely that adding links to large complex networks will often increase the chance that the enhanced network will include at least one simple network that has linktoll flexibility. This is not always true, since one could carefully add links with the specific aim to avoid additional flexibility. However, we conjecture that it is usually true when one adds links in some other manner, to an already complex network.

A detailed discussion of the probability of finding linktoll flexibility in large networks is beyond the scope of this present study. However, prior studies by Steinberg et al. [11, 12] indicate that paradoxical links (such as the Braess paradox of the bridge network class 3) are found more often than not in large networks. Since these simple-network parts of larger compound networks could bring their own link-toll flexibility, we conjecture that at least some link toll flexibility exists more often than not in large networks.

\section{ELASTIC DEMAND}

\subsection{Uniqueness of Path-Toll Solutions (Elastic Demand)}

Dafermos [1] claims on p.218: "It is clear that one may construct an infinite number of solutions $\mathrm{Rp}$ of the path-toll collection problem." This claim of non-uniqueness is true when user travel demand is fixed for each O/D pair. How- ever, we show here that it is not true for the more complicated, and more realistic, case of elastic demand.

When O/D demand is fixed, the procedure of adding a fixed toll to each path connecting this O/D pair will not change the overall O/D flow, and will not change the distribution among paths, thus allowing much flexibility in assigning path tolls. However, if the flow pattern is already system-optimized with elastic demand by using Marginal Cost pricing to maximize the sum of User Surplus and Toll Revenue [8], a uniform change in user-perceived cost for each path connecting an O/D pair, causes (by definition) an elastic change in the total O/D flow; thereby de-optimizing it. As an important limiting example, note that if demand is truly fixed, one can add equal tolls to each path ad infinitum, without affecting any flows. However, in any realistic case with elastic demand, it is clear that following the same procedure would eventually reduce the flows to zero; if the cost is sufficiently high, nobody can afford to make the trip.

Changing the toll of any used path would require a change in flow to readjust to the equilibrium, and would upset the optimality. Thus, one can not change costs of any used paths without upsetting the VSO conditions [13]. Furthermore, for the sake of completeness, we should note that decreasing the cost of an unused path to the cost level (or below) of a used path, would induce a flow on the previously unused path, thus again upsetting the optimal flows.

It is interesting to view the situation also from an economics viewpoint. It is well known that marginal cost (MC) pricing produces optimal flows and economic efficiency. Any additional uniform decrease/increase in path tolls, once MC pricing has been achieved, would be effectively a subsidy/tax and would tend to encourage over-use/under-use of the network. The case of perfectly inelastic demand is a very special case where a uniform decrease/increase in path tolls would not change the overall use (by definition of perfectly inelastic).

In principle, there could be some "degeneracies" that change the situation: such as e.g. non-monotone, multivalued demand-cost functions. However, except for such unlikely degeneracies, the flexibility in assigning the pathtolls while maintaining optimization under fixed demand, is totally lost under elastic demand.

In section 2, our original motivation and arguments were aimed at showing that there are many classes of networks for which planners can adjust link-tolls without affecting path flows. Somewhat ironically, here in section 3 we show that the flexibility provided to the link-toll planner in section 2 is now partially removed by the elasticity of user travel demand. Similarly, path-toll planners that had great flexibility under conditions of fixed demand, now have (except for occasional degeneracies mentioned above) no flexibility when the demand is elastic.

In practice, it may be possible to regain some of the lost path-toll flexibility. It turns out that the system cost improvement due to system-optimization is often a small percentage of the original user equilibrium system cost without any tolls. This is illustrated by the numerical example in our Table 1 for class 5, as well as various examples in the literature; e.g. Gartner [8], and the example by Penchina [9], and Arnott et al. [14]. Thus, although one can not maintain true 
optimal VSO conditions while changing path tolls under elastic-demand conditions, we see from examples that there may often be sufficient flexibility to make changes in pathtolls with only minor deviations from the overall VSO system costs. These minor deviations may be, in many cases, smaller than the inaccuracies of system models used to predict real life. The social gain obtained by these toll changes may sometimes outweigh the social harm caused by nonoptimal use of the network. A sensitivity analysis similar to that of Yang and Lam [6] would help to analyze such situations.

\subsection{Methods to Achieve Flexibility of Link-Toll Solutions: (Elastic Demand)}

We have seen in section 3.1 that, except for some possible degeneracies, changing path-tolls changes the network flows, by the simple definition of elastic demand. Thus, for elastic demand, path toll solutions are unique. On the other hand, depending on the details of the network, one may still have some flexibility to adjust link-tolls, so long as this is done in a way that keeps path-tolls constant. This is a stronger necessary condition (restriction) than we needed to provide link-toll flexibility under conditions of fixed demand where all path tolls had to change by the same amount.

Apparently, the full necessary and sufficient conditions for link-toll flexibility have not yet been investigated in general for elastic demands, and are beyond the scope of this work. We can, however, explain some specific ways to change link tolls while maintaining optimal flows in some of the network classes that were already introduced in section 2 .

\section{Elastic Demand - Class 1 from Section 2.1: Parallel}

In these disjoint networks, the path-toll Tij,k on any path $\mathrm{k}$ with multiple links can be kept constant (i.e. Tij,k = constant; $\mathrm{k}=1,2, \ldots \mathrm{K})$, while some link-tolls are increased and others decreased appropriately along the same path $\mathrm{k}$. Since the path-tolls are not changed, the flow pattern remains the same. See also discussion in Section 2.1.

\section{Elastic Demand - Class 2 from Section 2.2: Fixed Number of Links Per Path}

In these networks with fixed numbers of links per path, depending on how links are shared among paths, there may or may not be some paths for which it is possible to increase certain link-tolls and decrease others without changing any path-tolls. One simple example occurs if two links are shared by the same subset of paths; then one could increase the toll on the first link while decreasing the toll on the second by the same amount.

\section{Elastic Demand - Class 3 from Section 2.3: Bridge}

For this bridged network, the replacement of the two congestion tolls by a single larger bridge toll will eliminate the Braess paradox. However, it will change path costs, and hence the system flow when the demand is elastic. Any decrease/increase in path costs could be compensated by a positive/negative toll at the entrance to (or exit from) the network thereby achieving link-toll flexibility. If one insists on true link-tolls rather than entrance or exit tolls, one could expand the bridge network with a short un-congested link added in series with the entrance, and then apply the com- pensating positive/negative toll to this new link in the expanded bridge network.

\section{Elastic Demand - Class 4 from Section 2.4 Chain (Series)}

We can again provide a method (algorithm) to achieve link toll flexibility for this network. However, the restrictions on the flexibility are more severe. Consider again the simple example from Fig. (1, class 4) of a long chain of M monomers with a single origin at the beginning and a single destination at the end of the chain. Wherever only one link connects two nodes, one can only adjust that link-toll at will if one makes a compensating change elsewhere. One simple way would be to decrease the toll on another single link, or on each of another two-link pair, by the same amount as one increases the toll on this link.

Similarly, when two links connect two nodes, one can again add the same arbitrary (positive or negative) toll, but only if one compensates for it elsewhere. This compensation method could be the same as above. The compensation need not be done in this simple way, but could also be shared among many other links, so long as the total path toll remains the same. Hence, the restrictions on the link-toll planner are not very constraining, but are surely stronger than for the case of fixed demand.

When there are many origins and destinations at the junctions between monomers, one still has at least the flexibility of increasing/decreasing the toll on any single link (e.g. link c in Fig. (1, Class 4)), so long as one compensates it with a corresponding decrease/increase on each of the paired links (e.g. links a and b in Fig (1, Class 4)) within the same monomer, in order to keep all path tolls constant.

When there are many origins and destinations at the junctions within monomers (similar to those in concrete example of class 5 in Fig. 1), then the situation is more complicated. There is no simple procedure which allows changing link tolls for all such networks. In fact, if each link has one path using only that link, the link-toll solution is unique (see following discussion of class 5).

\section{Elastic Demand - Class 5 from Section 2.5 Concrete Ex- ample: Unique Link Tolls}

It is clear by examination that if there is a path that uses only a single link, then the toll on that link can not be increased without changing the path cost and consequently the elastic path flow.

Since each link in this example has a path which uses only that link, this restriction applies to all links, so this simple network has neither link toll flexibility nor path toll flexibility. The toll policy, link or path, for optimal flows under elastic demands is unique.

\section{Elastic Demand - Class 6 from Section 2.6: Combination of $1 \& 2$}

In this combination of classes 1 and 2 , it is clear that the link-tolls have the same flexibility as for class 2 . The combination neither adds nor removes any flexibility.

\section{Elastic Demand - Class 7 from Section 2.7. Compound}

Just as in the case of fixed demand, the compound network maintains at least the link-toll flexibility of the simple network added to the outer node. Again, we have no detailed 
knowledge of the probability for link-toll flexibility in general large networks. Clearly, the more restrictive conditions on unchanged (rather than equally changed) path tolls leads to more restricted link toll flexibility than for the case of fixed demand.

\section{DISCUSSION}

\subsection{Discussion (Fixed User Demand)}

For fixed user travel demand, link-toll and path-toll policies can be found which lead users to voluntarily choose an equilibrium flow pattern with tolls that replicate the systemoptimized flow pattern in the same network without tolls. The path-toll solutions were well known to be adjustable (non-unique). This gave planners great flexibility to adjust path-tolls in order to achieve additional (even non-traffic) social goals. Link-toll solutions were thought to be usually unique. Thus, although link-tolls may be easier to collect than path-tolls, it was thought that the uniqueness of the linktoll solutions prevented planners from using link-toll policies for additional goals.

We showed in section 2 that fixed-demand link-toll policies are much more flexible than previously thought. We illustrated several classes of networks for which link-toll solutions are not unique, even for large networks, and even if the number of paths greatly exceeds the number of links.

\subsection{Discussion (Elastic User Demand)}

The assumption that demand is fixed so that tolls can be raised indefinitely without changing flows seems naive. It might work well for short times in special situations with fairly low tolls that, even after they are increased, do not have a major effect on lifestyles. It may work when there are no convenient alternate routes. It does work fairly well (up to a point) in some analogous electric networks with electronically controlled constant current sources, and may also work well for a variety of different reasons for transportation of certain commodities such as milk, addictive drugs, guns, life-saving-medicines, etc.. Thus, the results of section 2 showing the non-uniqueness of link toll policies under fixed demand may have important consequences in many real networks too. However, in most real networks, one must deal with the elasticity of real user demands. Some recent work on elasticity of user demand can be found in the TDM online encyclopedia [15] and Cain et al. [16].

Having just demonstrated in section 2, a newly-found increased adjustability of link-tolls, and having just restated the well-known adjustability of path-tolls, we find the results of section 3 rather ironic. Elastic demand restricts the adjustability of link-tolls and essentially eliminates the flexibility of path-tolls. Thus, link/path-toll policies have almost switched the roles that they had under prior understandings of fixed demand: path-tolls usually unique and link-tolls sometimes adjustable under elastic demand.

Most real transportation situations have some elasticity in the user demand. This demand usually decreases/increases with corresponding increasing/decreasing perceived user costs, including time, distance, fuel, and tolls, as well as "costs" (positive or negative) such as weather, scenery, previous good or bad experiences, etc. Thus, traffic engineers who design tolls to achieve VSO in real networks, usually do not have the flexibility to adjust path-tolls without changing flows from their optimal levels. However, they often do have some flexibility to adjust link-tolls in order to achieve additional goals, while still achieving VSO. We have provided several examples with explanations for achieving this flexibility. Apparently, however, in a general network with elastic demand, the necessary and sufficient conditions for flexibility of link-tolls have not yet been determined.

Except for a brief mention by Penchina [9], the uniqueness of path-tolls under elastic demand has not been discussed in the literature. Even very recent works by Dial [4, 5] have not recognized this uniqueness of path tolls, or its consequent limitation on the flexibility of link tolls. This topic is discussed further by Penchina [7], who shows that minimal revenue (MR) pricing does not achieve optimal flows and economic efficiency when demand is elastic.

\section{CONCLUSIONS}

We have studied the uniqueness properties of toll policies that lead to Voluntary-System-Optimization (VSO) under conditions of fixed and elastic user demands.

Our present study shows that for fixed demand:

1. in agreement with previous work, there are multiple path-toll solutions; but

2. contrary to previous claims, there are also multiple link-toll solutions in many large complex networks.

Flexibility of toll policies for the case of elastic demand is discussed here for the first time. We find:

1. there is generally only one path-toll solution; but

2. there may still be multiple link-toll solutions (although fewer than for fixed demand).

We showed a complete turnaround in the flexibility available to path-toll and link-toll planners, compared to prior beliefs for fixed demand. Now, for elastic demand, path-tolls are essentially unique and link-tolls may have multiple solutions. So, with elastic demand, planners can no longer adjust path-tolls to achieve extra goals without upsetting the voluntary system optimization (VSO). However, they can sometimes achieve VSO and additional goals by adjusting link-tolls instead.

In any case, traffic models only approximate real traffic networks. Thus, the system administrator may be able to make changes in even "unique" path-tolls and link-tolls without deviating from VSO by more than the inaccuracies of the model. A system sensitivity analysis would be important to make these changes.

\section{ACKNOWLEDGEMENTS}

Early work on this project (May 1995) was presented as a term-paper in partial fulfillment of the requirements in Prof Anna Nagurney's course FOMGT341 "Logistics and Transportation", in the Dept of Finance and Operations Mgmt, at UMass/Amherst. I thank Prof Anna Nagurney for introducing me to the economics of toll policies for fixed demand. I am grateful to Prof David E Kaufman for helping me understand Dafermos [1]. I also thank Prof Michael Whiteman for 
suggesting this field of study. I wish to thank Prof Samuel S Bowles, Prof Herbert Gintis, Prof David Noyce, Prof Emily Parkany, and Prof James MacGregor Smith; I have enjoyed attending their lectures, where I learned a little about economics, game theory, transportation, and decision analysis. I also learned how closely these topics relate to one another, as well as to the biology, engineering, and physics, with which I was already more familiar. I have enjoyed and benefited from many interesting discussions with Prof Robin Lindsey, Prof James Banks, Prof. Kenneth Small, and more recently with Prof Benjamin Heydecker, and Dr. Robert Dial. I greatly appreciate editorial advice from Leslie Arriola and Leora Penchina, encouragement from Colette Penchina, Gil Penchina and Meira Penchina, and from Hyman Penchina who sought general solutions to broad inter-related social, economic, and scientific issues.

\section{ABBREVIATIONS}

$$
\begin{aligned}
\mathrm{MC}= & \text { Marginal Cost } \\
\mathrm{MR}= & \text { Minimal Revenue } \\
\mathrm{SO}= & \begin{array}{l}
\text { System Optimized (Minimizes social cost for } \\
\text { inelastic demand. For elastic demand, maximizes }
\end{array} \\
& \begin{array}{l}
\text { social surplus. Costs are minimized for any given } \\
\text { level of demand) }
\end{array} \\
\mathrm{UE}= & \text { User Equilibrium (each user minimizes his } \\
& \text { perceived costs) } \\
\mathrm{VSO}= & \text { Voluntary System Optimized } \\
\mathrm{O} / \mathrm{D}= & \text { Origin/Destination }
\end{aligned}
$$

\section{REFERENCES}

[1] S.C. Dafermos, "Toll patterns for multiclass-user transportation networks", Transport. Sci., vol. 7, pp. 211-223, 1973. Note: Equation 2.1 is based on proposition 1.1 on page 210 . The proof of this proposition had two errors, presumably typographical: (a) the second term in the un-numbered equation 7 lines below equations (1.10) is missing the superscript $\mathrm{i}$; (b) the un-numbered inequality in the final sentence of the proof could be corrected by replacing " $<0$ " by " > 0 ".

[2] A. Nagurney, Network Economics, Kluwer, Boston, 1993. See especially pp 154-156.

[3] A. Nagurney, Lecture Notes, FOMGT341, Logistics and Transportation, UMass./Amherst, MA 01003 USA (unpublished) 1995.

[4] R.B. Dial, "Minimal-revenue congestions pricing part I: A fast algorithm for the single-origin case". Transport. Res. B, vol. 33B, pp. 189-202, 1999.

[5] R.B. Dial, "Minimal-revenue congestion pricing part II: An efficient algorithm for the general case." Transport. Res. B, vol. 34B, pp. $209-228,2000$

[6] H. Yang, and W.H.K. Lam, "Optimal road tolls under conditions of queueing and congestion", Transport. Res. A, vol. 30A (5), pp. 319-332, 1996

[7] C.M. Penchina, "Minimal-revenue congestion pricing: some more good-news and bad-news", Transport. Res. B, vol. 38, pp. 559-570, 2004.

[8] N.H. Gartner, "Optimal traffic assignment with elastic demands: A review. Part I analysis framework" Transport. Sci., vol. 14, pp. 174-191, 1980. Note: example in Fig. (2B).

[9] C.M. Penchina, "Braess Paradox: Maximum penalty in a minimal critical network", Transport. Res. A, vol. 31A (5), pp. 379-388, 1997.

[10] M. Szwarc, Ionic Polymerization Fundamentals, Hanser: New York, 1996.

[11] R. Steinberg, and W.I. Zangwill, "The prevalence of Brass' Paradox", Transport. Sci., vol. 17, pp. 301-318, 1983.

[12] R. Steinberg, and R.E. Stone, "The prevalence of paradoxes in transportation equilibrium problems", Transport. Sci., vol. 22, pp. 231-241, 1988.

[13] N.H. Gartner, "Optimal traffic assignment with elastic demands: A review part II algorithmic Approaches" Transport. Sci., vol. 14, pp. 192-208, 1980

[14] R. Arnott, A. de Palma, and R. Lindsey, "Departure time and route choice for routes in parallel", Transport. Res. B, vol. 24B(3), pp. 209-228, 1990. Note examples in Fig. (3) and Tables 3 and 4.

[15] TDM Online Encyclopedia, "Transportation Elasticities: How Prices and Other Factors Affect Travel Behavior", http://www.vtp i.org/tdm/tdm11.htm, 2001.

[16] A. Cain, M.W. Burris, and R.M. Pendyala, "The impact of variable pricing on the temporal distribution of travel demand", TRB Paper Number 01-227, November 2000. Note: this is related to the Lee County Variable Pricing Project http://www.cutr.eng. usf.edu/its/ varprice.html 